\title{
MHD Free Convective Flow in a Composite Medium Between Co-axial Vertical Cylinders with Temperature Dependent Heat Flux on Inner Cylinder
}

\author{
Madhusudan Senapati ${ }^{1}$, Sampada K. Parida ${ }^{1}$, Bharat K. Swain ${ }^{2 *}$, Gouranga C. Dash ${ }^{1}$ \\ ${ }^{1}$ Dept. of Mathematics, Siksha "O” Anusandhan Deemed to be University, ITER, Bhubaneswar 751030, Odisha, India \\ ${ }^{2}$ Dept. of Mathematics, Indira Gandhi Institute of Technology, Sarang 759146, Odisha, India
}

Corresponding Author Email: bharatkeshari1@ gmail.com

https://doi.org/10.18280/mmep.070319

Received: 7 June 2020

Accepted: 16 August 2020

\section{Keywords:}

Brinkman extended Darcy model, free convection, heat flux, stress jump, magnetic field, composite medium

\begin{abstract}
The natural convective flow of conducting viscous fluid between two coaxial vertical cylinders partially filled with a porous material has been studied. The flow field is subjected to externally applied magnetic field (control input) and stress jump condition at the interface of two regions. The surface of the inner cylinder is subject to the constant heat flux and outer cylinder is maintained at constant temperature. The Brinkman extended Darcy model has been applied to porous media flow. The analytical solutions of the physical model are carried out with the help of modified Bessel function and numerical solutions by Runge Kutta method associated with shooting technique. The important findings are: the permeability of the medium and interface condition play vital role for the output of the desired flow rate and consistency of flow, the squeezing of the annular gap produces a cooling effect on cylindrical surfaces, the noticeable momentum transport occurs in the region close to the interface of fluid and porous region, the adjustable magnetic field (force-act-at-a distance) and stress jump condition (act-at-the contact) are to be simulated for obtaining desired smooth flow pattern.
\end{abstract}

\section{INTRODUCTION}

The severity of energy and ecology problems that confront us is a very good indicator for the interest in the buoyancy driven transport phenomena we call it natural or free convection. These flows arise simply because of the density variation caused by heat and mass transfer processes, in a body force field such as gravitational. Several processes of interest and importance such as nuclear reactor cooling system and underground energy transport are dominated by natural convective mechanisms. The classical problem of natural convective heat transfer from an isothermal heated vertical surface is assumed to be steady, laminar and the fluid properties, except density, are taken as constant. Based on Darcy's law and boundary layer approximation, Cheng and Minkowyez [1] obtained similarity solutions for the problem.

The fluid-flow and heat transfer in a composite system, partially filled with porous material, find numerous applications in thermal engineering pertaining to heat and mass transfer processes, oil extraction and heat exchangers etc. Many researchers such as Singh et al. [2], Ramanaiah et al. [3], Pop et al. [4] contributed to this field of research significantly using different fluid models. The flow was considered through porous media using Darcy / Brinkman models with different thermal conditions and geometrical configurations. Further, Paul and Singh [5] studied fully developed free convective flow between two coaxial vertical cylinders. Free convective flow for low-Prandtl-number fluid $(0.2<P r<1)$ in a horizontal annular region has been studied by Yoo [6]. The study leads to dual steady solutions when Rayleigh number exceeds a critical value.

The experimental studies also support some of the theoretical predictions. For example, flow through vertical and inclined elliptical tubes with constant heat flux has been experimented by Elshazly [7]. Further, experimental studies carried out by Seghir-Quali et al. [8] on an axial air flow with convective heat transfer inside a rotating cylinder and convective flow in a vertical circular cylinder with constant heat flux share the theoretical support of Mohammed and Salman [9].

A numerical study on buoyancy-driven unsteady natural convection boundary layer flow past a vertical cone embedded in a non-Darcian isotropic porous region with transverse magnetic field has been considered by Prakash et al. [10]. The transient fully developed free-convective flow of viscous incompressible fluid between two concentric vertical cylinders filled with porous material and saturated with the same fluid has been analysed by Jha et al. [11] when outer surface of inner cylinder is subject to isothermal or isoflux heating. Steady two dimensional MHD laminar free convective boundary layer flows of an electrically conducting Newtonian nanofluid over a solid stationary vertical plate in a quiescent fluid has been investigated numerically by Uddin et al. [12] with Newtonian heating at the bounding surface.

A finite element study of combined heat and mass transfer flow through a porous medium in a circular cylindrical annulus with Soret and Dufour effects in the presence of heat sources has been analysed by Gnaneswar [13]. The Thermo-Diffusion (Soret) and Diffusion - Thermo (Dufour) effects have been analysed by Reddy et al. [14] in a flow through porous annular region.

Further, The Darcy's law may be used as a basic equation for flow through porous medium, but it does not include the convective acceleration of the fluid. Therefore, this law is 
valid for low speed flow. The validity of law has been tested for wide domain of flows. For liquids, it is valid for small pressure differentials but in the case of liquids with high velocities or for gases at very low and at very high velocities, Darcy's law becomes invalid. The Reynolds number is the ratio of inertial to viscous forces. The use of Darcy's law assumes that the Reynolds number (based on pore size) is small for inertial effects to be unimportant. The Reynolds number for most ground water flows obey Darcy's law but flow with large gradient and/or flow where solid particles are large (coarse sand), the path of the fluid flow is curvilinear and the curvature of the path gives rise to inertial acceleration.

Thus, non-Darcy models incorporating the inertia due to high speed and the usual stress due to distortion velocity are proposed. In the present problem in Eq. (5), we have incorporated viscous resistances and inertia form to account for the non-Darcy factors. The Darcy flow model neglects the boundary and inertia effects on fluid saturated porous medium on the fluid flow and heat transfer. The simultaneous effects of fluid inertia force and boundary viscous resistance, upon flow and heat transfer with constant porosity in a porous medium, was studied by Vafai and Tien [15] for forced convection along a vertical plate in a fluid saturated porous medium. Both the boundary and inertia effects decrease the velocity and heat transfer rate in the boundary layer. The condition at the interface given in Eq. (5) takes care of both boundary viscous resistances as well as inertia effects on the flow of fluid. The speed of the fluid in the filter or the flow in the region where the velocity changes abruptly, are not always small and the convective force may be important. Brinkman [16] has proposed this convective force assuming that force on a particle situated in the cloud of particles could be calculated as if it were a solid particle embedded in a porous mass. Brinkman represented the porous mass by modifying Stokes equation adding Darcy resistance term to it so that the effect of all other particles is treated in an average basis and the resulting equation is a modification of Darcy's equation. By comparing with the experimental data the success of Brinkman's formula is indisputable. Tam [17] expressed Brinkman's method in better theoretical shape. The resulting generalized laws are found to be useful in the study of flow of highly porous media/porous materials such as foam metals and fibrous media usually have high porosity. For these high porosity media the boundary and inertia effects are important

If the flow arises naturally, simply due to the density difference, resulting from a temperature difference, in a body force field, such as gravitational field, the process is known as natural convection. The density difference gives rise to buoyancy effects for which the flow is generated. That is what the theme of the present problem. Thus, a natural interaction is established between the velocity and temperature fields, resulting coupling of momentum and energy equations. For example, a heated body cooling in ambient air generates such a flow in the region surrounding it.

In the free convection, the governing parameter is the Grashof number. This is a dimensionless number (ratio of buoyancy force and friction force). The Boussinesq approximation is related to the density difference, which causes the flow due to an interaction between the gravitational body force and the hydrostatic pressure gradient, can be expressed as a pure temperature effect as given by $\rho g B\left(T_{f}^{\prime}-T_{c}^{\prime}\right)$ in Eq. (1). $T_{f}^{\prime}$ and $T_{c}^{\prime}$ are fluid temperature and characteristic temperature or temperature of outer cylinder.

In the present analysis, the axisymmetry flow in a vertical concentric annuli has been considered. The flow between the cylinders is buoyancy driven. Let the axes of the cylinder be along $\mathrm{z}$-axis and $\mathrm{r}$ be the radial coordinate measured from the axis of the cylinder and $r$ be the radial coordinate measured from the outwards from the axis. The velocity components in the radial and tangential directions are zero and the velocity along axial direction is denoted by $\mathrm{u}$ and is only dependent on r. The pressure is considered to be constant [18]. The governing equation of the present flow problem with convection mode of heat transfer (free convection) and body force terms are given by Eqns. (1-4).

In the present flow model to account for the complicated processes, the non-Darcy Brinkman model [16] has been applied through which we have incorporated viscous resistances. Consequently stress due to distortion velocity in interface condition at $r^{\prime}=d^{\prime}$. We have restricted to low magnetic field consequently low magnetic Reynolds number, to neglect the induced magnetic field. The convective acceleration terms in the momentum equation have been neglected to do away with the non-linear terms. We have also used linear Boussinesq approximation. We have also assumed the medium is homogeneous and fully saturated. The flow is steady, laminar, incompressible and fully developed.

The novelty of the present study rests upon the flow characteristics of electrically conducting fluid subject to an externally applied magnetic field producing a body force actat-a distance and a stress jump condition act at-the-contact of the interface of composite medium with a constant heat flux at the surface of the inner cylinder. Further, the flow through saturated porous medium with uniform permeability has also been analysed by applying Brinkman extended Darcy model. Many industrial fluids and biological fluids are electrically conducting in nature. Therefore, an electrically conducting laminar fluid flow subject to transverse magnetic field finds application in MHD electrical power generator, MHD pump, electromagnetic flow meter and in extrusion of plastics in the manufacture of rayon and nylon etc. The stress jump conditions are of frequent occurrence in normal / abnormal biological systems.

The analytical solution by modified Bessel function has an edge over the error-bound numerical method. However, Runge-Kutta numerical method has also been applied to solve the equations for consistency and reliability of solutions. Both the methods applied in the analysis show a good agreement.

\section{FORMULATION OF THE PROBLEM}

Consider the problem of fully developed free convective flow in an annular region between two coaxial vertical cylinders in a composite medium i.e. clear fluid region and a saturated porous region. The flow is generated due to the difference in the temperature of the outer cylinder maintained at constant temperature and the inner one is subject to heat flux. In the present problem, the fluid is considered to be electrically conducting but the surfaces of the cylinders are nonconducting. A radial magnetic field of uniform strength $\left(0,0, B_{0}\right)$ has been applied, which produce a Lorentz force $\vec{J} \times \vec{B}=\sigma\left(\vec{E} \times \vec{B}-u B^{2}\right)$.

But $E=0, B^{2}=B_{0}{ }^{2}$, so $\vec{J} \times \vec{B}=\left(-\sigma_{f} u_{f} B_{0}{ }^{2}, 0,0\right)$. Therefore, since velocity components $u_{\theta}$ and $u_{z}$ are zero, the Lorentz force, a resistive force, having a single component acts perpendicular to the main direction of the flow. We assume 
that magnetic Reynolds number is small so that the induced magnetic field can be neglected in comparison with the applied one. This set up has also been shown in Figure 1.

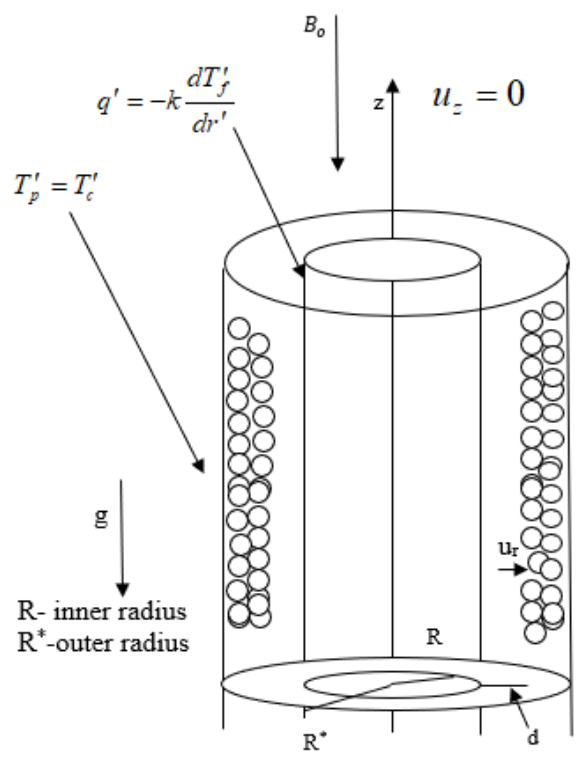

Figure 1. Flow geometry

Under the usual Boussinesq's approximation, the governing equations following [5] are given by:

Fluid region:

$$
\begin{gathered}
\mu_{f} \frac{d^{2} u_{f}^{\prime}}{d r^{\prime 2}}+\frac{\mu_{f}^{\prime}}{r^{\prime}} \frac{d u_{f}^{\prime}}{d r^{\prime}}-\sigma_{f} B_{0}^{2} u_{f}^{\prime}+\rho g \beta\left(T_{f}^{\prime}-T_{c}^{\prime}\right)=0 \\
\frac{d^{2} T_{f}^{\prime}}{d r^{\prime 2}}+\frac{1}{r^{\prime}} \frac{d T_{f}^{\prime}}{d r^{\prime}}=0
\end{gathered}
$$

Porous region:

$$
\begin{gathered}
\mu_{P} \frac{d^{2} u_{P}^{\prime}}{d r^{\prime 2}}+\frac{\mu_{P}^{\prime}}{r^{\prime}} \frac{d u_{P}^{\prime}}{d r^{\prime}}-\sigma_{p} B_{0}^{2} u_{p}-\frac{\mu_{f}}{K_{p}^{\prime}} u_{P}^{\prime}+\rho g \beta\left(T_{p}^{\prime}-T_{c}^{\prime}\right)=0 \\
\frac{d^{2} T_{P}^{\prime}}{d r^{\prime 2}}+\frac{1}{r^{\prime}} \frac{d T_{P}^{\prime}}{d r^{\prime}}=0
\end{gathered}
$$

The corresponding boundary conditions are

$u_{f}^{\prime}=0, \quad q^{\prime}=-k \frac{d T_{f}^{\prime}}{d r^{\prime}}$ at $r^{\prime}=R$

$u_{P}^{\prime}=0, T_{P}^{\prime}=T_{c}^{\prime} \quad$ at $r^{\prime}=R^{*}$

$\left.\begin{array}{l}u_{f}^{\prime}=u_{P}^{\prime}, \mu_{P} \frac{d u_{P}^{\prime}}{d r^{\prime}}-\mu_{f}^{\prime} \frac{d u_{f}^{\prime}}{d r^{\prime}}=\gamma \frac{\mu_{f}}{\sqrt{K^{\prime}}} u_{P}^{\prime} \\ T_{f}^{\prime}=T_{P}^{\prime}, \frac{d T_{f}^{\prime}}{d r^{\prime}}=\frac{d T_{P}^{\prime}}{d r^{\prime}}\end{array}\right)$ at $r^{\prime}=d^{\prime}$
Using the following non-dimensional quantities

$$
\begin{aligned}
& D a=\frac{K_{p}{ }^{\prime}}{R^{2}}, r=\frac{r^{\prime}}{R}, d=\frac{d^{\prime}}{R}, \lambda=\frac{R^{*}}{R}, u_{f}=\frac{u_{f}{ }^{\prime} v_{f} K}{g \beta q R^{3}}, \\
& u_{p}=\frac{u_{p}^{\prime} v_{p} K}{g \beta q R^{3}}, \theta_{f}=\frac{\left(T_{f}{ }^{\prime}-T_{c}{ }^{\prime}\right) K}{R q}, \theta_{p}=\frac{\left(T_{p}^{\prime}-T_{c}{ }^{\prime}\right) K}{R q} \\
& M_{f}=\sqrt{\frac{\sigma_{f} B_{0}{ }^{2} R^{2}}{\mu_{f}}}, M_{p}=\sqrt{\frac{\sigma_{p} B_{0}{ }^{2} R^{2}}{\mu_{p}}}, R v=\frac{\mu_{f}^{\prime}}{\mu_{p}^{\prime}}
\end{aligned}
$$

the Eqns. (1)-(4) become

$$
\begin{gathered}
\frac{d^{2} u_{f}}{d r^{2}}+\frac{1}{r} \frac{d u_{f}}{d r}-M_{f}^{2} u_{f}+\theta_{f}=0 \\
\text { and } \frac{d^{2} \theta_{f}}{d r^{2}}+\frac{1}{r} \frac{d \theta_{f}}{d r}=0 \text { (For fluid region } \\
R v \frac{d^{2} u_{p}}{d r^{2}}+\frac{R v}{r} \frac{d u_{p}}{d r}-\frac{1}{D a} u_{p}-M_{p}^{2} u_{p}+\theta_{p}=0 \\
\text { and } \frac{d^{2} \theta_{p}}{d r^{2}}+\frac{1}{r} \frac{d \theta_{p}}{d r}=0 \text { (Forporous region) }
\end{gathered}
$$

The boundary and matching conditions in dimensionless form are:

$$
\left.\begin{array}{l}
u_{f}=0, \quad \frac{d \theta_{f}}{d r}=-1 \quad \text { at } r=1 \\
\left.\begin{array}{l}
u_{p}=0, \quad \theta_{p}=0 \quad \text { at } r=\lambda \\
u_{f}=u_{p}, R v \frac{d u_{p}}{d r}-\frac{d u_{f}}{d r}=\frac{\gamma}{\sqrt{D a}} u_{p} \\
\theta_{f}=\theta_{p}, \frac{d \theta_{f}}{d r}=\frac{d \theta_{p}}{d r}
\end{array}\right\} \text { at } r=d
\end{array}\right\}
$$

The solutions of Eqns. (7)-(10) with boundary conditions (11) are given by

$$
\begin{gathered}
u_{f}=C_{1}+C_{2} \log r+\frac{r^{2}}{4}(\log r-\log \lambda-1) \\
u_{P}=C_{3} I_{0}\left(\frac{r}{\sqrt{R v D a}}\right)+C_{4} K_{0}\left(\frac{r}{\sqrt{R v D a}}\right)+D a(\log \lambda-\log r) \\
\theta_{f}=\theta_{P}=\log \lambda-\log r
\end{gathered}
$$

where, $I_{0}, K_{0}, I_{1}$, and $K_{1}$ are the modified Bessel functions of first kind and second kind of order zero and one respectively. 
Using Eqns. (12) and (13), the skin frictions on the walls at $r=1$ and $\lambda$ are calculated as:

$$
\begin{gathered}
\tau_{1}=-\left(\frac{d u_{f}}{d r}\right)_{r=1}=-C_{2}+\frac{1}{2}(\log \lambda+1)-\frac{1}{4} \\
\tau_{2}=-\left(\frac{d u_{f}}{d r}\right)_{r=\lambda}=-\frac{1}{\sqrt{R v D a}} C_{3} I_{1}\left(\frac{\lambda}{\sqrt{R v D a}}\right)+\frac{1}{\sqrt{R v D a}} C_{4} K_{1}\left(\frac{\lambda}{\sqrt{R v D a}}\right)+\frac{D a}{\lambda}
\end{gathered}
$$

\section{NUMERICAL INTEGRATION BY RUNGE-KUTTA METHOD}

For carrying out numerical integration, the Eqns. (7)-(10) are reduced to a set of first order differential equations. For this we make the following substitutions:

$$
\begin{gathered}
u_{f}=y_{1}, u_{f}^{\prime}=y_{2}, \theta_{f}=y_{3}, \theta_{f}^{\prime}=y_{4}, u_{p}=y_{5}, \\
u_{p}^{\prime}=y_{6}, \theta_{p}=y_{7}, \theta_{p}^{\prime}=y_{8}
\end{gathered}
$$

The reduced equations are

$$
\begin{gathered}
y_{2}^{\prime}=\frac{-1}{r} y_{2}+M_{f}^{2} y_{1}-y_{3} \\
y_{4}^{\prime}=\frac{-1}{r} y_{4} \\
y_{6}^{\prime}=\frac{1}{R v}\left\{\frac{-R v}{r} y_{6}+\left(\frac{1}{D a}+M_{p}^{2}\right) y_{5}-y_{7}\right\} \\
y_{8}^{\prime}=\frac{-1}{r} y_{8}
\end{gathered}
$$

Corresponding boundary conditions are given by

$$
\left.\begin{array}{c}
y_{1}=0, y_{4}=-1, y_{2}=?, y_{3}=? \text { at } \mathrm{r}=1 \\
y_{1}=y_{5}, R v y_{6}-y_{2}=\frac{r}{\sqrt{D a}} y_{5} \\
y_{3}=y_{7}, y_{4}=y_{8} \\
y_{5}=0, y_{7}=0 \text { at } \mathrm{r}=\lambda
\end{array}\right\} \text { at } \mathrm{r}=\mathrm{d}
$$

To start the integration, the values of $y_{2}$ and $y_{3}$ at $r=1$ are provided as guess values and the step by step integration is carried out from $r=1$ to $r=1.5$ then $r=1.5$ to $r=2$ with step length 0.01 using shooting technique with MATLAB code having error bound $10^{-6}$.

\section{RESULTS AND DISCUSSION}

The analytical and numerical solutions are obtained for the flow through a composite medium under the boundary and interface conditions and are presented through graphs and tables. The effect of applied transverse magnetic field producing a resistive force acting at a distance on the flow through an annular region is of special interest which has not been taken care of in the earlier studies.

Figure 2 shows the velocity distribution across the annular region for different values of magnetic parameter (magnetic Reynolds number) which determines the diffusion of magnetic field along the stream lines analogous to the ordinary Reynolds number for the diffusion of vorticity along the stream line, is small compared to the unity. On the other hand, when it is very large the magnetic field moves with the flow and is called frozen-in. In engineering problems, it is rare to obtain magnetic Reynolds number is greater than unity because of the low electrical conductivity of the useful fluids. From the figure it is seen that due to resistive force, the fluid velocity reduces in both fluid and porous regions. In this figure, the position of the interface is at $d=1.5$. In the porous region $(d>1.5)$, the velocity falls rapidly to attend the prescribed value. The maximum value of the profile occurs in the fluid region just before the transition zone.

Figure 3 shows the velocity profiles for different values of the Darcy number, characterizing the permeability of the porous medium. It is seen that as $D a$ increases, the velocity of the fluid increases under the dominating influence of magnetic field parameter of porous region over fluid region $\left(M_{p}=4\right.$ and $\left.M_{f}=2\right)$. On careful observation of curve $\mathrm{I}\left(D a=10^{-2}\right)$, it is seen that at the interface, fluidity of flow is detracted. Such effect is also marked in small measure when $D a=0.2$ and $D a=0.5$. Therefore, it is concluded that for maintaining smooth-flow across the flow domain, the right choice of Darcy number is essential.

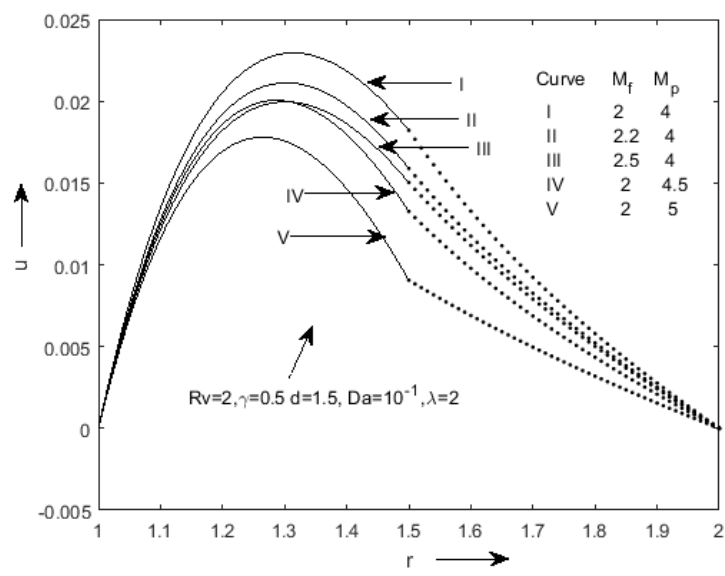

Figure 2. Velocity profile for various values of $M_{f}$ and $M_{p}$

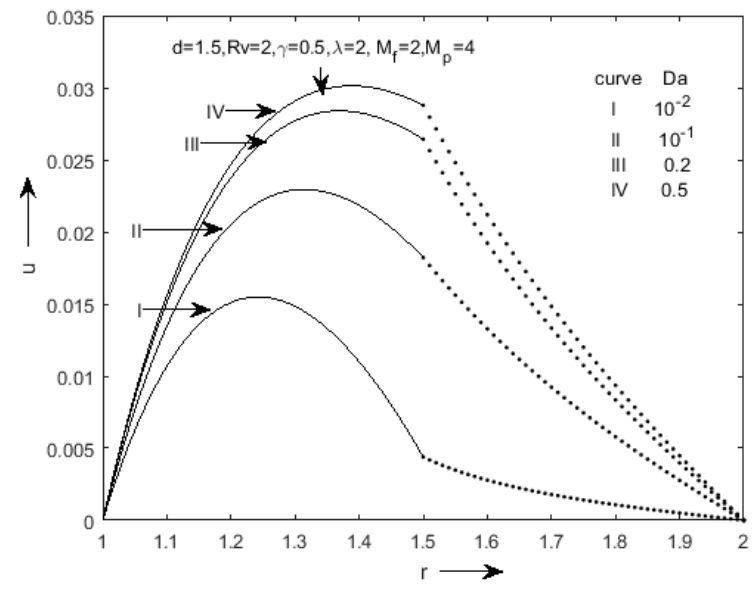

Figure 3. Velocity profile for various values of $D a$ 
Figure 4 depicts the velocity profile for various values of viscosity ratio $R v$ and it is observed that an increase in $R v$, decreases the velocity throughout the flow domain. Thus, it is concluded that under the influence of dominating effect of viscosity in fluid layer, momentum transport decreases and the decrease is significant in the layers adjacent to the interface $(d=1.5)$. The velocity becomes maximum when $R v=1$, i.e. both the viscosities are of the same order of magnitude. Further it is seen that as $\gamma$ increases, the velocity increases, whereas the effect of $R v$ is to decrease the velocity. The parameter $\gamma$ represents the adjustable proportionality coefficient of stress jump at the interface. The higher the jump, the greater the deformation, consequently, the velocity increases.

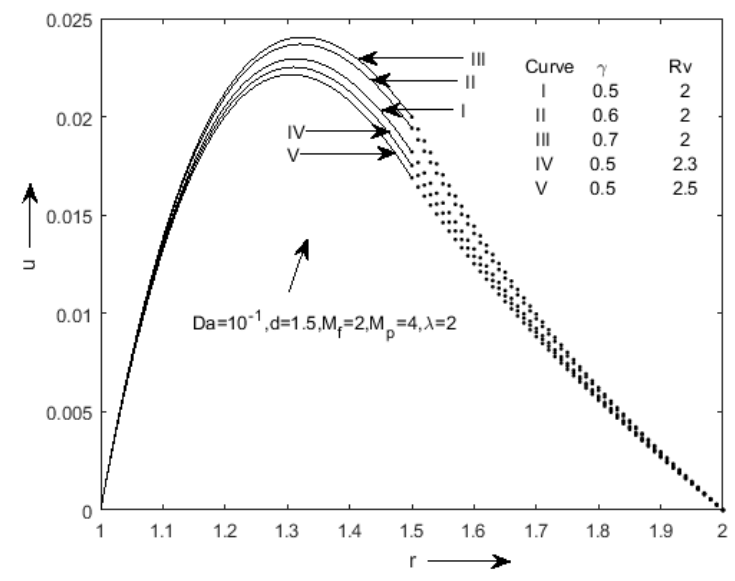

Figure 4. Velocity profile for various values of $\gamma$ and $R v$

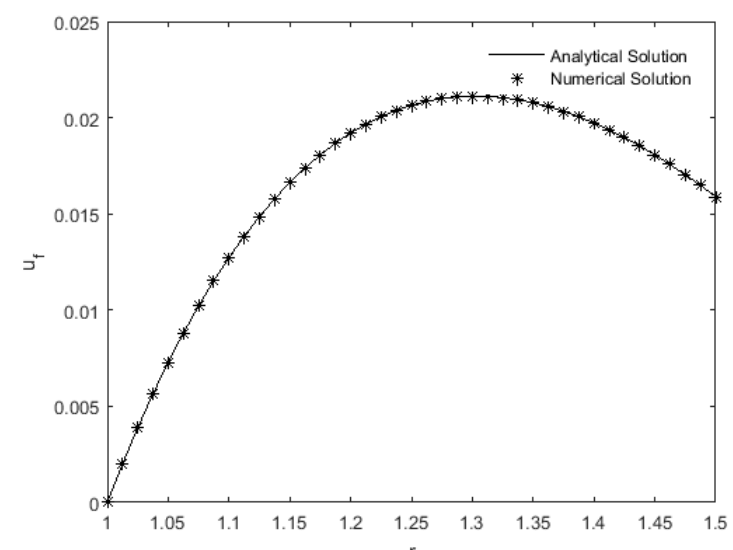

(a)

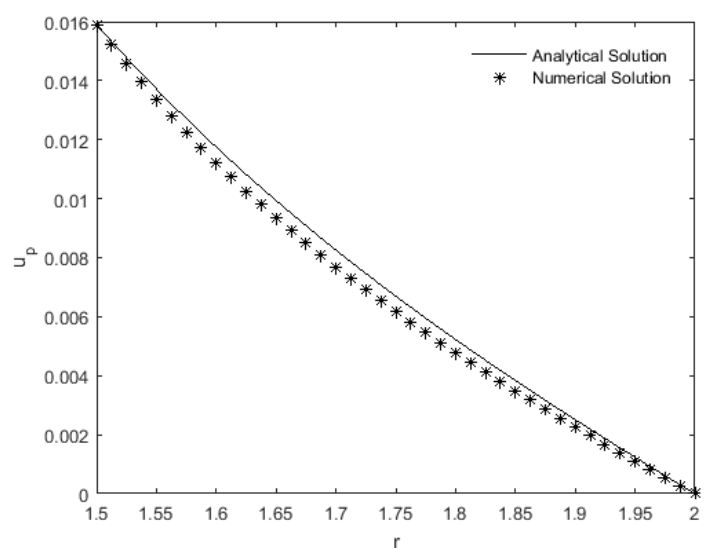

(b)

Figure 5. (a) Velocity in fluid region; (b) Velocity in porous region
We have verified our results with earlier published works (Kumari and Jayanthi [19]) and noticed that the results are found to be in good agreement. They have observed that both boundary (viscous resistances) and inertia effects are found to decrease the velocity in boundary layer. In the present study, from Figure 4 it is observed that an increase in viscosity ratio parameter $R v$ reduce the fluid velocity.

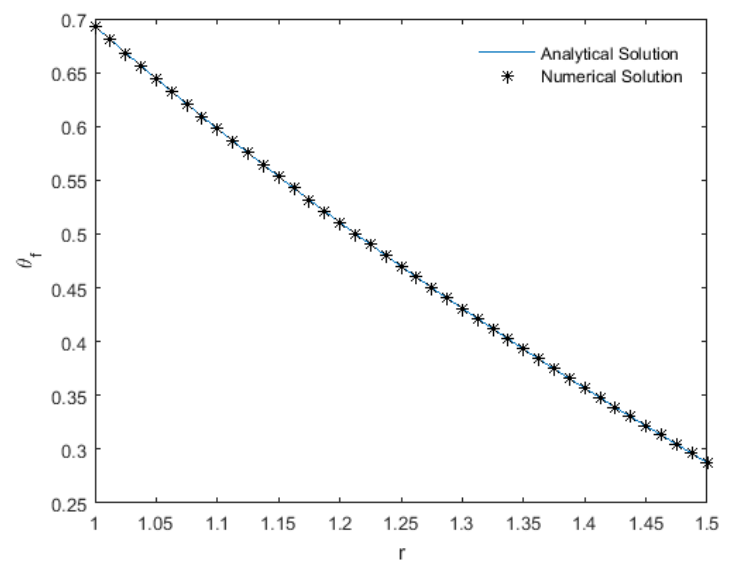

(a)

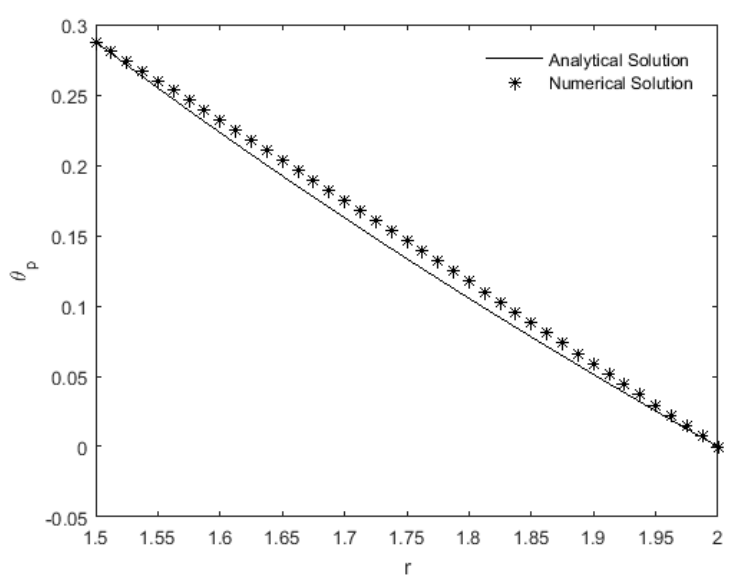

Figure 6. (a) Temperature in fluid region; (b) Temperature in porous region

Comparison between analytical solution and numerical solution when $R v=2, \gamma=0.5, D a=0.1, d=1.5, M_{f}=2.2, M_{p}=4, \lambda=2$ : Figure 5(a) and 5(b) show the velocity distributions in the fluid region $1 \leq \mathrm{r} \leq 1.5$ and in the porous region $1.5 \leq \mathrm{r} \leq 2$. Figure 6(a) and 6(b) represent the temperature distribution counterpart. From the Figures, it is seen that both analytic and numerical methods are in good agreement. It further asserts that velocity distribution assumes a parabolic distribution in the fluid region and stiff fall in porous region. It is also observed that sharp fall in temperature distribution is marked in both the regions. Those distributions adhere to no slip conditions and free stream at the bounding surfaces.

\section{CONCLUSIONS}

The significant momentum transport occurs in the region close to the interface of two zones.

The consistency of flow pattern depends on the permeability of porous region.

The strength of the external applied magnetic field is to be regulated to obtain the desired flow rate. 
The adjustable stress jump condition augments the velocity distribution.

The temperature distribution is almost linear across the flow domain and it decreases with the increase in spatial distance of annular region.

\section{ACKNOWLEDGMENT}

The authors are thankful to, Prof. Giulio Lorenzini, Editorin-Chief, MMEP and the reviewers for their kind suggestions for improvement of the manuscript.

\section{REFERENCES}

[1] Cheng, P., Minkowycz, W.J. (1977) Free convection about a vertical flat plate embedded in a porous medium with application to heat transfer from a dike. Journal $\mathrm{f}$ Geophysical Research, 82(14): 2040-2044. https://doi.org/10.1029/JB082i014p02040

[2] Singh, A.K. (1988). Natural convection in unsteady Couette motion. Defence Science Journal, 38(1): 35-41. https://doi.org/10.14429/dsj.38.4823

[3] Ramanaiah, G., Malarvizhi, G. (1990). Unified treatment of free and mixed convection on a permeable vertical cylinder in a saturated porous medium. Indian Journal of Technology, 28(10): 604-608.

[4] Pop, I., Cheng, P. (1992). Flow past a circular cylinder embedded in a porous medium based on the Brinkman model. International Journal of Engineering Sciences, 30(2): 257-262. https://doi.org/10.1016/00207225(92)90058-O

[5] Paul, T., Singh, A.K. (1998). Natural convection between coaxial vertical cylinders partially filled with a porous material. Forschung im Ingenieurwesen, 64: 157-162. https://doi.org/10.1007/PL00010772

[6] Yoo, J.S. (2003). Dual free-convective flows in a horizontal annulus with a constant heat flux wall. International Journal of Heat and Mass Transfer, 46(13): 2499-2503. 9310(02)00539-2

https://doi.org/10.1016/S0017-

[7] Elshazly, K., Moawed, M., Ibrahim, E., Emara, M. (2005). Heat transfer by free convection from the inside surface of the vertical and inclined elliptic tube. Energy Conversion and Management, 46(9-10): 1443-1463. https://doi.org/10.1016/j.enconman.2004.07.003

[8] Seghir-Quali, S., Saury, D., Harmand, S., Phillipart, O., Laloy, D. (2006). Convective heat transfer inside a rotating cylinder with an axial air flow. International Journal of Thermal Sciences, 45(12): 1166-1178. https://doi.org/10.1016/j.ijthermalsci.2006.01.017

[9] Mohammed, H.A., Salman, Y.K. (2007). Combined natural and forced convection heat transfer for assisting thermally developing flow in a uniformly heated vertical circular cylinder. International Communication in Heat and Mass Transfer, 34(4): 474-491. https://doi.org/10.1016/j.icheatmasstransfer.2007.01.00 1

[10] Prakash, J., Mohiddin, S.G., Varma, S.V.K. (2013). Free convective MHD flowpast a vertical cone with variable heat and mass flux. Journal of Fluids, 2013: 1-9. https://doi.org/10.1155/2013/405985

[11] Jha, B.K., Yusuf, T.S. (2016). Transient free convective flow in an annular porous medium: A semi-analytical approach. Engineering Science and Technology, an International Journal, 19(4): 1936-1948. https://doi.org/10.1016/j.jestch.2016.09.022

[12] Uddin, M.J., Khan, W.A., Ismail, A.I. (2012). MHD free convective boundary layer flow of a nanofluid past a flat vertical plate with Newtonian heating boundary condition. PLOS One, 7(11): e49499. https://doi.org/10.1371/journal.pone.0049499

[13] Gnaneswar, K. (2014). Effect of thermo-diffusion and chemical reaction on mixed convective heat and mass transfer through a porous medium in cylindrical annulus with heat source. International Journal of Engineering Research and Applications, 4: 1-16.

[14] Reddy, P.S., Rao, V.P. (2012). Thermo-diffusion and diffusion -thermo effects on convective heat and mass transfer through a porous medium in a circular cylindrical annulus with quadratic density temperature variation - finite element study. Journal of Applied Fluid Mechanics, 5(4): 139-144.

[15] Vafai, K., Tien, C.L. (1981). Boundary and inertia effects on flow and heat transfer in porous medium. International Journal of Heat and Mass Transfer, 24(2): 195-203. https://doi.org/10.1016/0017-9310(81)90027-2

[16] Brinkman, H.C. (1947). A calculation of viscous force exerted by a flowing fluid on a dense swarm particle. Appl. Scient. $\quad$ Res., 1 1: 27-34. https://doi.org/10.1007/BF02120313

[17] Tam, C.K.W. (1969). The drag on a cloud of spherical particles in low Reynolds number flow. Journal of Fluid Mechanics, 38(3): 537-546. https://doi.org/10.1017/S0022112069000322

[18] Schlichting, H., Gensten, K. (2000). Boundary Layer Theory. $8^{\text {th }}$ edition, Springer, 116-117.

[19] Kumari, M., Jayanthi, S. (2008). Non-Darcy mixed convection flow of non-Newtonian fluids on a vertical surface in a saturated porous medium. International Journal of Fluid Mechanics Researches, 35(5): 459-474. https://doi.org/10.1615/InterJFluidMechRes.v35.i5.50

\section{NOMENCLATURE}

$d$

$D a$

$d$

$\mathrm{g}$

$K_{p}^{\prime}$

$M_{f}$

$M_{p}$

$Q$

$R$

$R^{*}$

$R v$

$T_{c}^{\prime}$

$T_{f}^{\prime}$

$T_{p}^{\prime}$

$u^{\prime}$

$U$ radial distance of interface, $m$

Darcy number

radial distance of interface in nondimensional form, $\mathrm{m}$ acceleration due to gravity, $\mathrm{m} . \mathrm{s}^{-2}$ permeability of the porous medium

magnetic parameter in fluid region magnetic parameter in porous layer rate of heat transfer radius of the inner cylinder radius of the outer cylinder viscosity ratio parameter characteristic temperature, $\mathrm{K}$ temperature of the fluid layer, $\mathrm{K}$

temperature of the porous layer, $\mathrm{K}$

velocity along the axis of cylinder, $\mathrm{m} . \mathrm{s}^{-1}$ velocity along the axis of cylinder in nondimensional form, $\mathrm{m} . \mathrm{s}^{-1}$ 


\section{Greek symbols}

$\alpha$ adjustable coefficient in the stress jump condition coefficient of thermal expansion, $\mathrm{K}^{-1}$ ratio of dynamic viscosity dynamic viscosity, $\mathrm{kg} \cdot \mathrm{m}^{-1} \cdot \mathrm{s}^{-1}$ temperature in non-dimensional form, $\mathrm{K}$ 